Revista Electrónica Complutense de Investigación en Educación Musical ISSNe: 1698-7454

https://dx.doi.org/10.5209/reciem.62101

\title{
Gestión del aula inclusiva a través del proyecto LÓVA: la ópera como vehículo de aprendizaje ${ }^{1}$
}

\author{
Aida Sanahuja Ribés²; Odet Moliner García ${ }^{3}$; Lidón Moliner Miravet ${ }^{4}$
}

Recibido: 28 de octubre de 2018 / Aceptado: 11 de abril de 2019

Resumen. Con este trabajo se pretenden analizar las implicaciones que tiene el proyecto LÓVA en la gestión del aula inclusiva. Se ha utilizado una metodología cualitativa, centrada en el estudio descriptivo de un caso. Los instrumentos de recogida de la información han sido: la observación científica, la entrevista y el análisis documental. El análisis de contenido se ha realizado mediante la herramienta ATLAS.ti. Los resultados describen cómo se ha llevado a cabo el proyecto LÓVA en un aula de $5^{\circ}$ y $6^{\circ}$ de primaria de un colegio rural agrupado de la provincia de Valencia (España). Así mismo, se extraen aquellos elementos que están presentes en el proyecto LÓVA y nos permiten avanzar hacia la construcción de aulas más inclusivas. Concretamente, se detecta cómo en el proyecto LÓVA se parte del reconocimiento a la diversidad y se lleva a cabo una diferenciación de las estructuras, del contenido, el proceso y del producto. También, se ha identificado cómo y en qué momentos se favorece una cultura colaborativa, un liderazgo compartido y una participación más democrática en el proyecto. A modo de conclusión cabe decir que a través del proyecto LÓVA se fomenta una gestión inclusiva en el aula.

Palabras clave: Proyecto LÓVA; Educación Inclusiva; Educación Musical; Gestión del Aula; Estudio de Caso.

\section{[en] Inclusive classroom management through the project LÓVA: the opera as a vehicle for learning}

\begin{abstract}
The aim of this study is to analyze the implications that the project LÓVA has in the management of inclusive classrooms. A qualitative methodology has been used, based on a descriptive case study. The tools used for data collecting were scientific observation, interviews and document analysis. Data analysis has been conducted using the software ATLAS.ti. The results of this study describe how the project LÓVA has been implemented in a classroom of 5th and 6th grade of Primary school in a Gathered Rural School in the Province of Valencia (Spain). Furthermore, there have been identified those elements which are present in the project LÓVA and which allow us to advance towards the development of more inclusive classrooms. Specifically, it is identified how in the project LÓVA we start from the recognition of diversity, and then a differentiation is made in the structures, the content, the process and the product. In addition, it has also been identified how and when a collaborative culture, a shared leadership and a more democratic participation in the project is fostered. In conclusion, inclusion is promoted in the classroom management through the project LÓVA.
\end{abstract}

1 Este artículo forma parte de un trabajo más amplio enmarcado en una ayuda para contratos predoctorales para la formación de doctores contemplada en el Programa Estatal de Promoción del Talento y su Empleabilidad, en el marco del Plan Estatal de Investigación Cientifica y Técnica y de Innovación 2013-2016 (Referencia: BES2014- 068165). Financiado por el MINECO y el FSE.

2 Universitat Jaume I

E-mail: molgar@uji.es

$3 \quad$ Universidad de Valencia

E-mail: molgar@uji.es

$4 \quad$ Universitat Jaume I

E-mail: mmoliner@uji.es 
Keywords: project LÓVA; Inclusive Education; Musical Education; Classroom Management; Case Study.

Sumario. 1. Introducción, 1.1. El proyecto LÓVA: la ópera como vehículo de aprendizaje. 1.2. Hacia una gestión más inclusiva del aula. 2. Método. 2.1. El caso. 2.2. Procedimiento e instrumentos para la recogida de datos. 3.3. Procedimiento de análisis de datos. 3. Resultados. 4. Discusión de los resultados y conclusiones. 5. Referencias

Cómo citar. Sanahuja Ribés, A.; Moliner García, O.; Moliner Miravet, L. (2019). Gestión del aula inclusiva a través del proyecto LÓVA: la ópera como vehículo de aprendizaje. Revista Electrónica Complutense de Investigación en Educación Musical, 16, 3-19.

\section{Introducción}

\subsection{El proyecto LÓVA: la ópera como vehículo de aprendizaje}

LÓVA es un proyecto educativo que se desarrolla en centros escolares con la idea de que una clase pueda transformarse en una compañía para crear su propia ópera. Ese es el pretexto para aprender en equipo, puesto que se plantea como un reto que entre todos los estudiantes van a tener que superar. Los estudiantes se convierten en protagonistas de su propio aprendizaje organizándose en profesiones y realizando tareas que van, desde escribir el libreto, confeccionar el vestuario o los decorados, hasta su representación. Su principal valor es, en palabras de Sarmiento (2012a), el desarrollo de la identidad a través de las artes escénicas, la música, el trabajo en equipo y la superación de desafíos, creando un aula como espacio abierto al riesgo, las emociones y la experimentación.

Según indica Joy (2002), el proyecto LÓVA surge a partir de un proyecto educativo que Taylor y Forman fundaron hacia los años 70 en la Ópera de Seattle y que en los 80 se extendió al Metropolitan Opera Guild (Nueva York). El proyecto comenzó en España (en Madrid) en el año 2006, gracias a la norteamericana Mary Ruth McGinn, la cual efectuó la adaptación del proyecto Creating Original Opera deEE. UU. (Pérez-Aldeguer, 2013). Ante la gran acogida, en 2008 se formalizó LÓVA como proyecto educativo entre la fundación SaludArte y el Teatro Real de Madrid, bajo la coordinación de Pedro Sarmiento. Desde el año 2016, docentes de todos los niveles educativos realizan cursos de formación para poder implementar este proyecto en las aulas de los centros en los que desarrollan su labor docente.

El proyecto va más allá de la educación musical y las artes escénicas, ya que la música, junto a las diferentes disciplinas o áreas (lengua, matemáticas, plástica o educación física, entre otras), genera conexiones que dotan de significado al proyecto. Así pues, LÓVA es un arte que necesita la aportación de casi todas las disciplinas y oficios que ponen su empeño en articular un espectáculo escénico llevado a cabo a lo largo de un curso escolar en el que alumnado, docentes y otros agentes participan, crean y trabajan para representar una ópera infantil (Gil, 2017). El alumnado adopta un rol activo a lo largo de todo el transcurso del proyecto, incluso en la gestión y la toma de decisiones:

Todo está decidido, creado y gestionado por niños: el libreto, la iluminación, la escenografía, el nombre de la compañía y su logotipo, el trabajo actoral, la publicidad, la compra de materiales, la música y su ejecución instrumental, el vestuario, el 
maquillaje, la utilería, la regiduría, la gestión del presupuesto, la coordinación de los trabajos, etc. (Sarmiento, 2012a, p.41).

Tal y como muestran algunas investigaciones (Llopis, Roselló y Villarroya, 2009; Pérez-Aldeguer, 2010, 2013), la música influye en la idea de aula como comunidad y fomenta el sentido de pertenencia, pues es un elemento con una clara repercusión en la cohesión del grupo y la inclusión social de sus miembros.

La música, por ser un lenguaje universal, es un medio para la creación de espacios de paz (Kent, 2008), ha servido como vehículo para transformar conflictos que existían entre personas (Cabedo, 2014a) y puede servir también para dar respuesta a los nuevos retos que van surgiendo tanto en las aulas como en las sociedades (Cabedo, 2014b). De hecho, desde la antigua Grecia, ha sido considerada como un componente que favorece al desarrollo integral de la persona, dado que ayuda no solo en el desarrollo de las capacidades artístico-creativas, sino también a la integración sociocultural del individuo (Pérez y del Basto, 2011). En consecuencia, LÓVA favorece el desarrollo personal a través del empoderamiento y la autonomía del alumnado (Sarmiento, 2012b), lo cual es un elemento digno de estudio en relación a la gestión de la diversidad de las aulas escolares.

\subsection{Hacia una gestión más inclusiva del aula}

Una escuela que pretende ser inclusiva exige al docente una especial sensibilidad hacia el hecho de que todo el alumnado tiene diferencias, necesidades y capacidades variadas (Prud'homme, Vienneau, Ramel y Rousseau, 2011). Cuando una institución educativa adopta un modelo inclusivo, tanto los docentes como el resto de miembros de la comunidad, comparten una serie de valores basados en el respeto, el trabajo cooperativo, el diálogo, el valor de las diferencias y la justicia social (Rojas y Haya, 2018). Es entonces cuando cobra especial relevancia la idea de la escuela como comunidad educativa vinculada a la responsabilidad compartida y al sentido de pertenencia, pues:

Muchas escuelas y clases inclusivas que consiguen su meta y enfatizan la comunidad se centran en el modo de organizarse, de tal manera que todos se sientan ligados, aceptados y apoyados, y en las que cada uno apoya a sus compañeros y a los demás miembros de la comunidad, al tiempo que se satisfacen sus necesidades educativas. (Stainback, Stainback y Jackson, 1999, p.23)

Junto a la promoción de valores inclusivos y al sentido de comunidad, surge otro de los propósitos de la educación inclusiva que, según apunta Arnaiz (2003), radica en la consideración de la necesidad de que todos los estudiantes reciban una educación acorde a sus necesidades individuales. Esta idea coincide con la de Stainback y Stainback (1999), quienes subrayan que las aulas inclusivas se caracterizan por el reconocimiento y la gestión de la diversidad, con el objetivo de que los miembros del aula reciban una educación conforme a sus características, a la vez que se incrementan las posibilidades de aprendizaje para todas y todos. Por tanto, la diversidad no se percibe como una dificultad, sino que más bien es entendida como un fabuloso recurso para propiciar el aprendizaje de todo el alumnado en el aula (Moriña, 2008; Booth y Ainscow, 2011).

Los trabajos de Tomlinson $(2005,2008)$ aportan algunas claves que facilitan dar respuesta a las necesidades de todo el alumnado en el aula ordinaria, partiendo de la idea de que el alumnado presenta diferentes intereses, aptitudes y estilos de aprendizaje. Esta autora introduce el concepto de "aula diferenciada" retomando la idea de diferenciación de la enseñanza (Sanahuja, 2017). Siguiendo a Perrenoud (1996), ésta consiste en actuar 
de modo que cada estudiante se halle, lo más a menudo posible, en situaciones de aprendizaje productivas para él. Este concepto, desde una visión inclusiva y en términos de una perspectiva de enseñanza, consiste en la capacidad del docente de reconocer, valorar y tomar parte de la diversidad de una situación pedagógica que contribuye al aprendizaje individual y colectivo. Además, favorece el reconocimiento de las similitudes y de las diferencias en el seno del grupo (intercomprensión) y en un proyecto compartido de educación para todos (interdependencia) (Prud'homme, Dolbec y Guay, 2011). La enseñanza diferenciada es, sobre todo, una forma de pensar sobre la enseñanza, el aprendizaje y la evaluación, una filosofía que guía todas las prácticas educativas y una manera de poner en valor las diferencias y aprovecharlas. Consiste, según Kline (2015) en incorporar estrategias de enseñanza para atender a las necesidades, los estilos de aprendizaje y los intereses de los estudiantes. Según indica Bergeron (2015), la diferenciación pedagógica requiere de una gestión de la diversidad basada en una lógica proactiva. Ésta precisa de una planificación de la enseñanza en función de la diversidad del alumnado y de sus necesidades, opuesto a la lógica reactiva que consiste en planificar la enseñanza para el alumnado medio e intervenir en el alumno que presenta mayor dificultad. Esto está en consonancia con los principios de la educación inclusiva, concebida como un proceso que permite responder a la diversidad de las necesidades de todos los educandos a través de una mayor participación en el aprendizaje, las actividades culturales y comunitarias y reducir la exclusión dentro y fuera del sistema educativo (UNESCO, 2005, p.14). Para trabajar bajo los parámetros de la inclusión en el aula, los docentes plantean sus propuestas didácticas diferenciando: las estructuras (Caron, 2003, Leroux y Paré, 2016), el contenido, el proceso y el producto (Caron, 2003; Tomlinson, 2005; Levy, 2008; Kline, 2015; Leroux y Paré, 2016; Wan, 2017).

En la tabla 1, se presenta una breve descripción de cada uno de los elementos a diferenciar.

Tabla 1. Principales elementos a diferenciar en el aula inclusiva.

\begin{tabular}{|c|l|}
\hline \multicolumn{2}{|c|}{ DIFERENCIACIÓN PEDAGOGÍA DESDE UNA PERSPECTIVA INCLUSIVA } \\
\hline LAS ESTRUCTURAS & $\begin{array}{l}\text { Elementos que están directamente relacionados con la organización del aula } \\
\text { como: agrupaciones, modalidades de trabajo, tiempo, espacios y los recursos } \\
\text { materiales y/o personales (Caron, 2003; Leroux y Paré, 2016). }\end{array}$ \\
\hline EL CONTENIDO & $\begin{array}{l}\text { El contenido es aquello que un alumno debe llegar a conocer, comprender y ser } \\
\text { capaces de hacer como resultado de una experiencia de aprendizaje (Tomlinson, } \\
\text { 2008). Asimismo, también engloba a todos los medios que ponen al alumno en } \\
\text { contacto con la información objeto de ser aprendida, por ejemplo: variedad de } \\
\text { textos, saberes y conocimientos, el grado de complejidad o profundidad y los } \\
\text { diferentes materiales didácticos (Leroux y Paré, 2016). }\end{array}$ \\
\hline EL PROCESO & $\begin{array}{l}\text { El proceso incluye cómo los docentes enseñan (las actividades que ofrecen deben } \\
\text { de abordar las diferentes capacidades del alumnado) y cómo los alumnos aprenden } \\
\text { (Levy, 2008). Por tanto, se diferencia el proceso según el campo de interés del } \\
\text { alumnado, los conocimientos previos y el nivel de competencias, las retroalimen- } \\
\text { taciones entre el alumno y el docente o el alumno y sus pares, nivel de desarrollo } \\
\text { del alumnado (motor, cognitivo, social...), motivación y compromiso hacia la tarea } \\
\text { y el modo de vida del alumnado (Leroux y Paré, 2016). }\end{array}$ \\
\hline $\begin{array}{l}\text { Alude a la manera en que los alumnos demuestran los aprendizajes que han ad- } \\
\text { quirido (Caron, 2003; Levy, 2008). O sea, las diferentes modalidades de expre- } \\
\text { sión, los destinatarios, los proyectos o creaciones, las modalidades de evaluación } \\
\text { del aprendizaje, así como, los criterios y niveles de aprendizaje (cualitativos o } \\
\text { cuantitativos) (Leroux y Paré, 2016). Tomlinson (2008), lo entiende como el ve- } \\
\text { hículo mediante el cual el alumno muestra lo que ha comprendido y sabe hacer } \\
\text { como resultado de un aprendizaje. }\end{array}$ \\
\hline PRODUCTO
\end{tabular}


Otro aspecto fundamental para el aula inclusiva y que atañe a la convivencia escolar, se refiere al clima del aula y a las interacciones que se producen entre sus miembros. El aula se entiende como un espacio de intercambio de conocimientos y experiencias en el que tienen que tener cabida la diversidad de todo el alumnado (Masip y Rigol, 2000). En la misma línea, Arnaiz (2005) apunta que educar para la diversidad se sostiene en una convivencia democrática donde la cooperación, la solidaridad y la tolerancia tienen que regir y predominar en las interacciones entre todo el alumnado. De este modo se deben de fomentar «aulas saludables» entendidas como un espacio en el que los estudiantes se sientan aceptados, apoyados, ayudados y seguros (Tomlinson, 2008). Aulas donde se celebra la diversidad y se crea el sentido de comunidad (Stainback y Stainback, 1999).

El principal propósito de este trabajo es abordar las implicaciones que tiene el proyecto LÓVA (la ópera como vehículo de aprendizaje) en la gestión de un aula inclusiva de educación primaria. Concretamente, en el contexto de este estudio, nos preguntamos: ¿cómo se ha llevado a cabo el proyecto LÓVA en un aula de $5^{\circ}$ y $6^{\circ}$ de educación primaria? y ¿cuáles son los diferentes elementos y componentes propios del proyecto LÓVA que ayudan a la gestión del aula inclusiva? A partir de las mismas, formulamos los dos objetivos específicos de la investigación: a) Describir el desarrollo del proyecto LÓVA en el aula, y b) Identificar los elementos esenciales del proyecto relacionados con la gestión del aula inclusiva.

\section{Método}

El trabajo se enmarca en una metodología cualitativa, utilizando el estudio descriptivo de un caso como estrategia de investigación. Según indica Stake (1998), el estudio de caso se centra en la complejidad, la exclusividad, la singularidad y la particularidad. Esta investigación referente al proyecto LÓVA se ha llevado a cabo en un aula multinivel de un colegio rural agrupado (CRA) durante un curso escolar. Así pues, acotamos el aula como unidad de estudio.

\subsection{El caso}

El aula objeto de estudio está formada por un total de 21 alumnos, 7 de $5^{\circ}$ de primaria y 14 de $6^{\circ}$ de primaria de edades comprendidas entre 10 y 12 años. Se trata de un aula multinivel en la que la tutora plantea su docencia incorporando estrategias para la inclusión de todo el alumnado, como el aprendizaje cooperativo o la tutoría entre iguales. El proyecto ha sido propuesto e impulsado por la maestra especialista en educación musical, con formación previa en el proyecto LÓVA. La colaboración de la maestra tutora ha jugado un papel muy importante a lo largo de todo el proyecto puesto que ha coordinado su docencia con la dinámica del proyecto para un abordaje interdisciplinar de las materias que impartía. También participaron las familias de los alumnos del aula, así como otros agentes externos a la comunidad escolar (por ejemplo, ayuntamiento o auditorio).

\subsection{Procedimiento e instrumentos para la recogida de datos}

Para cumplir con los requisitos éticos de la investigación se procedió a firmar con la tutora un consentimiento informado (Sandín, 2003) en el que quedaban recogidas 
las principales acciones del proyecto de investigación. La dirección del centro fue informada y, al tratarse de menores, se solicitaron las autorizaciones a los tutores del alumnado.

Con el propósito de llevar a cabo una triangulación de los datos y de las fuentes, se acudió a la utilización de diferentes instrumentos, técnicas y voces para la recogida de la información (Okuda y Gómez-Restrepo, 2005; Aguilar y Barroso, 2015). Así pues, la recogida de datos se ha realizado mediante la observación no participante, la entrevista y el análisis de material.

Para las observaciones se ha utilizado una hoja registro de observación (Prud'homme, LeBlanc y Paré, 2013), la cual se focaliza en los siguientes aspectos: 1) rigor y coherencia entre las intenciones, los contenidos y las actividades pedagógicas; 2) anticipación (planificación) y toma en consideración de la diversidad; 3) prácticas de enseñanza y de evaluación; 4) gestión y clima del aprendizaje y 5) recursos atribuidos para efectuar adaptaciones, modificaciones y ofrecer un apoyo particular. Se han tomado notas de campo, que han sido apoyadas a partir de la grabación en vídeo y fotografías.

Por otro lado, a través de la entrevista se han obtenido informaciones complementarias a la observación (Simons, 2011), así como múltiples visiones del caso objeto de estudio (Stake, 1998). Además, se confeccionó un guión de entrevista semiestructurada diferente para cada uno de los colectivos entrevistados (maestras, alumnado y familias) sobre el desarrollo del proyecto, su implicación y valoraciones. Se han realizado un total de 11 entrevistas, dos a las docentes, una a una madre y ocho al alumnado, con objeto de obtener información complementaria para indagar sobre sus valoraciones que han sido grabadas y transcritas literalmente.

Algunos ejemplos de preguntas realizadas fueron:

a) a la maestra de música (p.ej.: “¿Has recibido alguna formación sobre cómo llevar a cabo el proyecto LÓVA en el aula?, ¿Con esta distribución por oficios se responde a las habilidades, necesidades e intereses del alumnado?"),

b) a la maestra tutora (p.ej.: "¿Cuál ha sido tu papel en el proyecto LÓVA?, ¿Cómo te has coordinado con la docente de música?, ¿Cómo valoras el proyecto LÓVA en relación a los aprendizajes efectuados por el alumnado?"),

c) al alumnado (p.ej.: “¿Cómo os han ayudado las familias en el proyecto LÓVA?, ¿De qué manera hicisteis la elección y distribución de los oficios de la ópera?”) y

d) a una madre (p.ej.: “¿Cómo se os informó de que se estaba llevando a cabo el proyecto LOVA en el aula de vuestros hijos?, ¿En qué aspectos se centró la participación o colaboración de las familias en el proyecto LÓVA?, ¿Cómo valoras el proyecto LÓVA en relación a los aprendizajes del alumnado?”).

En cuanto al análisis documental (Ruiz-Olabuénaga, 2012), se han seleccionado materiales de diversos tipos producidos para el desarrollo didáctico del proyecto, tanto por las maestras como por el alumnado, como esquemas de los oficios, mapas conceptuales, logos, carteles, resúmenes de los actos y fotografías.

\subsection{Procedimiento de análisis de datos}

Recogida toda la información, se procedió a la transcripción literal de las entrevistas, se generó un proyecto mediante el software ATLAS.ti al que se incorporaron el resto 
de documentos a analizar y se procedió al tratamiento de los datos mediante análisis de contenido. Se procedió a la reducción de datos mediante la codificación en unidades de sentido. En un primer análisis se realizó una codificación temática de carácter deductivo a partir de tres categorías teóricas descritas en la literatura como factores de inclusión: 1) reconocimiento y gestión de la diversidad mediante la diferenciación pedagógica, 2) clima de aula que genere una convivencia democrática y 3) sentido de pertenencia a la comunidad. En un segundo nivel de análisis emergieron las subcategorías. Se asignó un sistema de códigos con el objetivo de identificar en cada momento la procedencia del dato (Ver Tabla 2).

Tabla 2. Sistema de códigos para la identificación de la procedencia de los datos.

\begin{tabular}{|c|c|}
\hline Instrumento & Informante \\
\hline Entrevista (E) & Madre (M) \\
\hline Observación (O) & Alumnado (A) \\
\hline Notas de campo (NC) & Docente Tutora (D) \\
\hline Análisis del material (AM) & Docente música (Dm) \\
\hline
\end{tabular}

Con objeto de representar gráficamente la relación entre las categorías se confeccionó un mapa relacional mediante el programa CmapTools.

\section{Resultados}

Se presentan los resultados en función de los dos objetivos de la investigación.

a) Describir el desarrollo del proyecto LÓVA en el aula.

\section{Inicio}

El proyecto LÓVA consiste en crear una ópera infantil cuyo propósito principal es que todo el alumnado trabaje para conseguir un objetivo compartido.

Dentro de la ópera, cada una de las profesiones es muy importante para el objetivo final, que es la producción final de esa ópera. Desde el director hasta el técnico de luces, hasta el vestuario, maquillaje, documentalista... cualquiera de las profesiones es súper importante [E_Dm].

En un primer momento, el alumnado formó una compañía de ópera llamada $D A C A$ $P O$ y se comprometieron con el proyecto: “... ellos firman también como un contrato de que están de acuerdo, de que quieren formar una compañía de ópera” [E_Dm].

La maestra de música cuenta que efectuó el curso junto a un compañero que ya había realizado diferentes cursos de danza con ella.

[...] estuve el verano pasado [...] haciendo el curso con él. Y me gustó mucho. Tenía mucho miedo a hacerlo porque claro, nosotros hicimos un curso muy ex- 
press, de 4 o 5 días, nada más, y este curso pienso que necesita al menos un par de semanas más para ya consolidar más aspectos [E_Dm].

Así pues, el proyecto LÓVA se inició por iniciativa de la maestra de música con la colaboración de la tutora. "Ella es la que daba las instrucciones a principio de curso [...], yo estaba más en la sombra" [E_D].

\section{Desarrollo}

Temporalmente, este proyecto se realiza durante todo el curso escolar, principalmente en la asignatura de música. Aunque también se trabaja en la asignatura de plástica y la docente tutora integra algunos contenidos en otras asignaturas.

Es que está todo ligado en este proyecto. Entra el lenguaje, entra la música, entran las matemáticas, entra la plástica, entra todo. Lo que pasa es que por currículum pues a veces no puedes coger todas las sesiones que quieres [E_Dm].

En un primer momento, el alumnado va tomando un primer contacto con cada una de las profesiones necesarias para llevar a cabo una ópera.

[...] durante todo el primer trimestre, ellos investigan las profesiones, viene gente a contarles. Yo les paso unos documentos. Y ellos van recopilando información. El objetivo del escritor ¿Cuál es? ¿Te gustaría ser escritor? Y hay varias preguntas. A ver, escribe el principio de un cuento en el que aparece una bruja. Son pequeñas actividades y preguntas personales, para yo saber hacia dónde van orientados sus gustos [...] [E_Dm].

Antes de las fiestas de Navidad y una vez conocidas las profesiones, se da a escoger a cada alumno los tres oficios que les gustaría desempeñar. "Y ellos ponen por orden de preferencia, la que más les gusta la $1 .^{\mathrm{a}}$, después la 2. ${ }^{\mathrm{a}}$ y después la $3 .^{\mathrm{a}}$. Para intentar darles la que a ellos más les gusta y más cómodos se puedan sentir" [E_Dm].

La ópera estuvo escrita antes de Pascua y para ello los escritores tuvieron que trabajar duro en coordinación con el director y el regidor para poder terminarla a tiempo y con la ayuda de la maestra.

Los escritores, que eran los que más a contrarreloj iban porque el regidor y el director les dijeron que la obra tenía que estar escrita antes de Pascua, que después tenían que ensayar. En Pascua, quedaron conmigo algunos días de fiesta para que les ayudara en alguna cosa. Y los guionistas tuvieron también que aceptar críticas [E_Dm].

\section{Resultado}

La obra se representó en el centro educativo y en el auditorio de la localidad. "El día 12 de junio, en el Auditorio del pueblo" [E_A2]. Pero antes, el ensayo general “...aquí en la escuela lo hacemos el día 26" [E_Ā 1]. "E1 26, una ópera sólo para que la vean los alumnos y otra para todos" [E_A2]. 
b) Identificar los elementos esenciales del proyecto relacionados con la gestión del aula inclusiva.

Seguidamente, en la Figura 1, se presentan los diferentes elementos y dimensiones del proyecto LÓVA articulados en relación a la gestión del aula inclusiva.

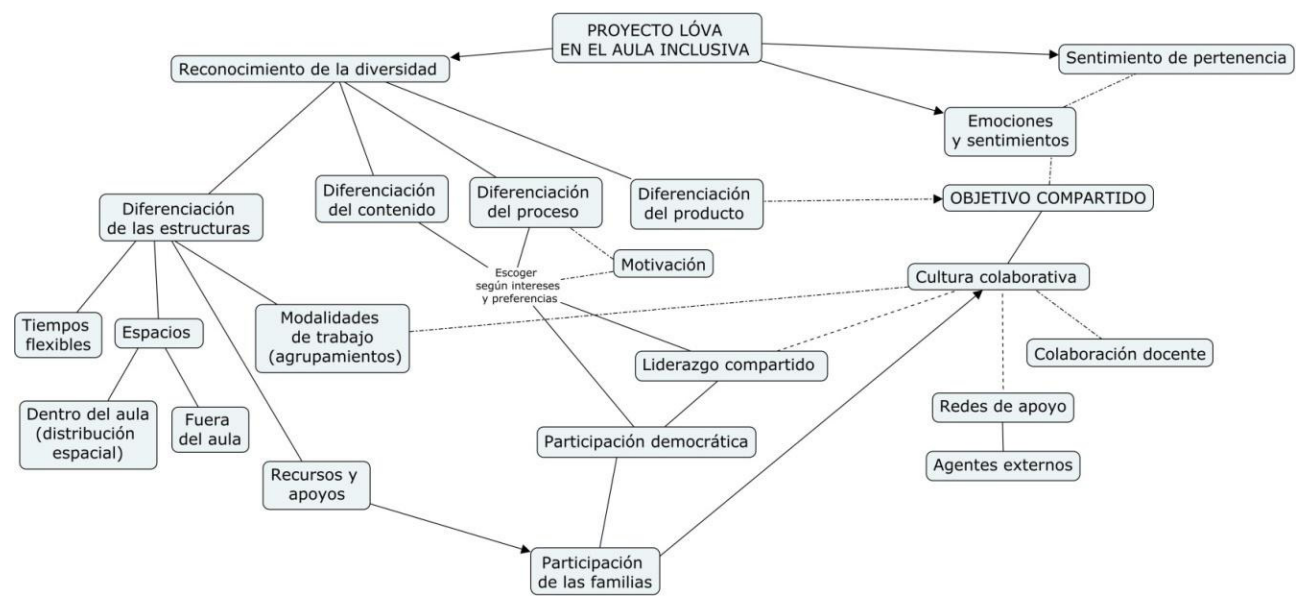

Figura 1. Mapa relacional de los principales elementos del proyecto LÓVA en la gestión del aula inclusiva.

Fuente: Elaboración propia.

Reconocimiento de la diversidad y diferenciación pedagógica

En el proyecto LÓVA se parte del reconocimiento de la diversidad del alumnado presente en el aula y se indaga sobre sus preferencias e intereses y por ello escogen según sus gustos, el rol o profesión que más le interesa.

"Primero nos enseñaron en qué consisten las diferentes faenas y después ya hemos escogido [...] las que más nos gustaban y las maestras nos han dado las tareas que pensaban que..." [E_A2]. "Y nosotros teníamos que marcar tres y ella intentaría que uno de esos tres se cumpliera" [E_A8].

En cada una de las profesiones hay dos o tres alumnos trabajando para cumplir el objetivo marcado por el grupo aula. En el segundo trimestre los roles ya están distribuidos, y cada uno de los alumnos empieza a trabajar en aquellas competencias que son de su principal interés. Por tanto, existe una diferenciación del contenido y del proceso, el cual viene mediado por la tarea que debe desempeñar cada alumno o grupo de alumnos para trabajar en miras de conseguir el objetivo colectivo que se ha marcado toda la clase. Además, hay que destacar que la diferenciación del contenido está presente también en la temática escogida para abordar la ópera, dado que ésta surge de los intereses e inquietudes del alumnado.

Los roles que se deben desempeñar para llevar a cabo la ópera fueron: director, regidor, documentalista, relaciones públicas, escritor, músicos, actores, escenografía, vestuario y maquillaje. A continuación, se pasan a describir cada uno de los roles citados: 
a) El director y el regidor eran profesiones separadas, pero debían coordinarse y trabajar muy estrechamente.

El regidor, se encargaba de que todo estuviera organizado, cada uno de los trabajos y sobre todo se encargaba de ensayar con los intérpretes. Se iban dando trucos, consejos de cómo tenían que hacer las cosas. El director, que era el que tenía que hacer las instancias, pedir permiso al auditorio, tenía que animar a los compañeros [...] [E_A6].

b) El documentalista y relaciones públicas también trabajaban más conjuntamente.

Yo tengo que hacer entrevistas a cada miembro de la compañía y colgar las entrevistas y lo que está pasando cada día en el blog de la clase [E_A6].

Uno se encargaba más de hacer los carteles, tickets, dípticos, o trípticos, diseñar el logotipo, ya finalmente como quedaría. Documentalista, se encargaba más de hacer las entrevistas a sus compañeros, de hacer noticias, de ir escribiendo en el blog de la clase, en qué momento se encontraban, que estaban haciendo, buscar periódicos que les publicaran o llamar a la radio [E_Dm].

c) La alumna perteneciente al grupo de los escritores explica que tuvieron que escribir el guión de la ópera.

Pues yo lo que hago es: primero escribimos en un papel el guión de la ópera, después lo revisamos, lo pasamos al ordenador, lo volvimos a revisar, lo imprimimos y ahora estamos con los intérpretes para ayudarles a cómo hacer los gestos y todo eso [E_A5].

d) Los músicos compusieron e interpretaron la música de todos los actos de la ópera.

Los músicos eran los compositores. Durante todo el curso han hecho de compositores y el día de la obra hicieron de músicos. Hacen las propias canciones y ellos decidian si lo tocaban en el clarinete, si lo tocaban en el teclado, si cogían la flauta, si la persona que sabía tocar la trompa tocaba la trompa. Ellos se lo habían dividido [E_Dm].

e) Había 5 actores que representaron y cantaron la ópera.

f) Los técnicos de luces y escenografía trabajaron colaborativamente.

Técnicos de luces y sonido eran dos niños que trabajaban conjuntamente. Porque claro, el técnico de luces y de sonido hasta la semana o dos semanas antes que no podían ir a probar el sonido ni nada. Por tanto, han trabajado durante todo el primer y segundo trimestre con los escenógrafos, que solamente eran dos y necesitaban mucha ayuda [E_Dm].

g) También había niños encargados del maquillaje y el vestuario. "Después teníamos maquillaje y vestuario, teníamos uno de maquillaje y uno de vestuario, pero trabajaban paralelos" [E_Dm]. 
Se detecta como a través de la diferenciación del proceso (escoger roles o profesiones según su interés o motivación), las docentes buscan un eje motivador para desarrollar el proyecto y la maestra extrapola dicha temática en las actividades de aula. Emerge entonces una categoría que denominamos liderazgo compartido, visto que cada grupo de alumnos adopta unas funciones u oficios con el objetivo de conseguir un mismo objetivo compartido por todos los integrantes del aula. Además, este liderazgo se comparte entre las docentes pues la tutora, desde un principio, sigue todo el proyecto en el aula y ello permite la integración disciplinar de los contenidos del currículo según el Real Decreto 126/2014, de 28 de febrero, por el que se establece el currículo básico de la Educación Primaria.

Está muy bien que estés dentro del aula, porque después ella (hace referencia a la maestra de música) pide ayuda en cuanto a otras áreas. Pues, por ejemplo, ella quiere trabajar la descripción de los personajes, por tanto, tú ya sabes que en castellano en la descripción... Aprovechas lo que este proyecto te está pidiendo [E_D]. [...] pues mira ahora vamos a hacer los personajes, trabaja los adjetivos. Ahora vamos a trabajar la descripción del lugar. [...] Nos vamos coordinando para que ella también en lenguaje vaya trabajando esas cosas. O después, por ejemplo, las matemáticas a la hora de dibujar utilizar la escala. [...] o a la hora de medir, trabajamos los metros, los decímetros, los centímetros... [E_Dm].

También hay una diferenciación de las estructuras. Los tiempos y los espacios son flexibles. Mientras en el 1. ${ }^{\text {er }}$ trimestre del proyecto toda la clase trabaja más al unísono, en el $2 .^{\circ}$ y $3 .^{\text {er }}$ trimestre la diferenciación de los tiempos es simultánea, ya que encontramos a cada alumno o grupo de alumnos trabajando en una tarea específica. Esta organización requiere utilizar diferentes espacios de trabajo tanto dentro como fuera del aula.

N. está trabajando en un ordenador, está preparando un díptico. Los escenógrafos también están trabajando, unos trabajan sobre el cartel en el pasillo y otros trabajan en el decorado en clase. Los músicos están ensayando en el patio junto a los actores [O_NC].

Cada subgrupo de profesiones u oficios aborda unos objetivos concretos a desarrollar que desembocan en una diferenciación del producto. La suma de las aportaciones de cada grupo de oficios o profesiones hacen posible el éxito del producto final.

\section{Clima de aula que genere una convivencia democrática}

Durante todo el primer trimestre se trabaja mucho a nivel de grupo y a nivel emocional. "[...] trabajamos mucho los sentimientos, sus relaciones, ¿Qué sienten?, ¿Qué les pasa?, ¿Qué les preocupa?” [E_Dm]. A partir de esas emociones se hacen ciertas preguntas que son las que les conducirán a delimitar y escribir el tema de la ópera. "Por ejemplo, había preguntas sobre: ¿qué cosas nos hacen felices?, ¿qué cosas nos da miedo?, ¿qué cosas nos preocupa?, ¿qué cosas nos hace estar tristes? ...” [E_Dm].

Además, todas las decisiones son tomadas y acordadas por el alumnado que, en diferentes momentos del proyecto, van negociando y decidiendo sobre los diferentes aspectos que competen al diseño, planificación y puesta en práctica de la ópera, lo que incide en el fomento de la participación democrática. 
[...]las decisiones debian tomarlas los alumnos [...]. Evidentemente, P. (hace referencia a la maestra de música) tampoco tomaba la decisión de autoridad, porque el proyecto es eso: el alumno piensa eso y tienes que hablar con ellos, pero las decisiones son de ellos [E_D].

Las familias también ayudan: "Al principio de curso nos hicieron una explicación de que la maestra de música llevaba adelante todo un proyecto que se llamaba LÓVA., [...] que es un proyecto de ópera" [E_M1]. Una vez el alumnado tuvo todos los aspectos concertados y decididos, se dieron cuenta que necesitaban ayuda de sus familiares. "[...] éramos nosotros los que teníamos que crear sin ayuda de los padres, sólo nosotros, después cuando ya sabíamos lo que íbamos a hacer ya nos ayudaron, por ejemplo, en escenografía" [E_A2].

[...] ellas mismas dijeron: ¿bueno necesitáis ayuda? y escenografía, 'si, nosotros si'. A escenografía les costó bastante porque tuvieron que hacer un autobús gigante [...] Y dibujar en grandes dimensiones, [...] vinieron bastantes padres a ayudar en la parte de escenografía [...] [E_Dm].

Generalmente, el alumnado valora positivamente la participación de las familias en el aula. "A mí personalmente me gusta mucho que puedan interactuar los padres en la clase, que los padres puedan venir a ayudar en lo que haga falta" [E_A1]. También se contó con algunos extras. "Necesitamos personas de $3 .^{\circ}$ y $4 .^{\circ}$ que sólo van a salir, pero de relleno" [E_A5].

Además, otro elemento que emerge son las redes de apoyo, en la medida en que la maestra de música se refuerza y se apoya en compañeros que hicieron el curso con ella y desarrollan el proyecto en sus aulas. El profesional que impartió el curso de formación se comprometió a llevar un seguimiento a los docentes que se reunían mensualmente para compartir experiencias, sensaciones y trazar algunas propuestas de mejora.

Creamos un grupo de WhatsApp que todavía continua. '¡Ay!, es que necesito que venga un documentalista. ¿Conocéis a algún periodista?' Y eso se iba reforzando, se iba creando una ayuda. Eso vino muy bien [E_Dm].

Una de las familiares entrevistadas también puso de manifiesto la gran responsabilidad que supuso para el alumnado emprender un proyecto de gran envergadura.

A lo mejor se sienten un poco estresados porque claro, es una responsabilidad [...] Tienen un poco de miedo de ver cómo les sale [E_M1].

Sentido de pertenencia al aula y a la comunidad

El trabajo de las emociones y sentimientos que se van generando incide también en el sentido de pertenencia. El proyecto LÓVA ayudó considerablemente a consolidar el grupo clase y fortaleció los lazos y vínculos de los diferentes miembros del aula. 
Y cuando terminaron el abrazo que se hicieron entre ellos, cómo lo disfrutaron, y organizaron una cena todos juntos. Yo creo que sí, que unió mucho al grupo, que es un poco la finalidad... que sean compañeros y saber trabajar todos juntos [E_Dm].

La noticia de la representación fue publicada en los medios locales, gracias a las gestiones efectuadas por la documentalista y la ópera fue representada al final de curso en el Auditorio de la localidad afianzando el sentimiento comunitario.

Tras trabajar de manera cooperativa y coordinada, los resultados del proyecto fueron muy satisfactorios, de ahí que emerge otro factor de la inclusión como es la cultura colaborativa.

El proyecto puede salir o no salir. La finalidad es que, si todos han trabajado en equipo, todos han trabajado juntos, han sabido compartir, han sabido decirse las cosas, han sabido organizarse, quererse, al final el trabajo sale. Y salió [E_Dm].

Finalizado el proyecto, unos alumnos fueron junto a la maestra de música al Palacio de las Artes y las Ciencias de Valencia a contar su experiencia a los docentes que estaban realizando el curso de formación:

Fuimos con tres niños y alli disfrutaron mucho. Les cantaron las canciones y se sintieron como muy premiados, estuvo bien. Y en este caso el palacio de las artes en Valencia les hizo una visita y les enseñaron todas las profesiones y espacios de una ópera [E_Dm].

\section{Discusión de los resultados y conclusiones}

En cuanto a la descripción de cómo se ha llevado a cabo el proyecto LÓVA en el aula objeto de estudio, los resultados apuntan a la importancia de la implicación del alumnado y del profesorado en el desarrollo del proyecto (Sanahuja, 2017). El alumnado, de acuerdo con los trabajos previos de Sarmiento (2012a) cobra un papel protagonista durante todo el proceso, asumiendo compromisos, tomando decisiones y trabajando por un objetivo común: llegar a representar una ópera. Es un trabajo arriesgado, complejo y que requiere, de acuerdo con Gil (2017) la aportación de varias disciplinas, además de la música. Esto permite abordar de manera interdisciplinar diferentes áreas curriculares y promueven un aprendizaje funcional y significativo que origina niveles más altos de motivación del alumnado hacia la tarea (Reeve, 1996).

Con el desenlace del proyecto se llega a articular un espectáculo que, de acuerdo con Sarmiento (2012b) favorece el desarrollo personal, el empoderamiento y la autonomía del alumnado.

Respecto de los elementos del proyecto LÓVA que favorecen una gestión de la diversidad en el aula inclusiva, los resultados del caso analizado nos permiten recoger, a modo de conclusiones, las siguientes:

a) Se reconoce la diversidad y se favorece la diferenciación pedagógica ofreciendo una enseñanza acorde a las necesidades, intereses y ritmos de aprendizaje 
del alumnado (Tomlinson, 2008; Kahn, 2010; Sanahuja, 2017). Si nos centramos en los indicadores que caracterizan la diferenciación de las estructuras (Leroux y Paré, 2016), se ha visto la flexibilidad de los tiempos (Bernal, 2007) y la variedad de los espacios, ofreciendo una diferenciación simultánea tanto dentro como fuera del aula (Aylwin, 1992) que ayuda al alumnado a gestionar los espacios y a optimizar el tiempo de trabajo (Jorba y Cassellas, 1997). La modalidad de trabajo imperante en cuanto a la relación y gestión de los recursos o apoyos personales, nos aproxima a una idea de cooperación (Johnson, Johnson y Holubec, 1999; Pujolàs, 2008). Se favorece la ayuda ofrecida por los iguales mediante la configuración de pequeños grupos de oficios en los que todos los alumnos participan y trabajan para conseguir un mismo objetivo compartido: articular la ópera.

b) Se genera un clima de aula «saludable» (Arnaiz, 2005; Tomlinson, 2008) trabajando de manera explícita emociones y sentimientos, lo cual permite que los estudiantes se sientan aceptados, ayudados y seguros para afrontar el gran reto del aprendizaje. Esto es muy importante para la gestión del aula inclusiva y favorece una participación democrática en el sentido en que Apple y Beane (1999) proponen como oportunidad de vivir la democracia en la escuela. A ello contribuye también la ayuda ofrecida a través de la colaboración y participación de las familias de manera que se genera una vía de participación e implicación familiar en el centro, como la que Epstein (2001) denomina «colaboración con el entorno», puesto que hace referencia a las relaciones que el centro educativo establece con el contexto sociocultural próximo, apoyado por las familias. Además, se han generado redes de apoyo entre los docentes que realizaron la formación y llevaron a cabo el proyecto en sus respectivas aulas (Ugarte, 2007).

c) Se desarrolla el sentido de pertenencia al grupo aula, uno de los factores clave del proyecto repercute en el compañerismo y en el clima, aspecto esencial para propiciar un aula inclusiva. A ello contribuyen tanto los elementos ya mencionados en relación al reconocimiento de la diversidad y al clima de aula, como sin duda la música que, de acuerdo con Pérez-Aldeguer (2013) y Llopis et al. (2009) fomenta la cohesión del grupo y la inclusión social de sus miembros.

En síntesis, podemos decir que, se ha observado que el proyecto se basa en elementos que favorecen la educación inclusiva. Es necesario realizar investigaciones que exploren esta misma línea en diferentes centros de diferentes países. Esta investigación podría servir como ejemplo de las implicaciones que tiene implementar proyectos que favorezcan la educación inclusiva en las aulas, lo cual podría servir de inspiración para otros docentes.

\section{Referencias}

Aguilar, S. y Barroso, J. (2015). La triangulación de datos como estrategia en investigación educativa. Revista de Medios y Educación. 47, 73-88. Recuperado de https://recyt.fecyt. es/index.php/pixel/article/view/61672

Apple, M. W. y Beane, J. A. (1999). Escuelas democráticas. Madrid, España: Morata. Arnaiz, P. (2003). Educación Inclusiva, una escuela para todos. Málaga, España: Aljibe. 
Arnaiz, P. (2005). Atención a la diversidad. Programación curricular. San José, Costa Rica: EUNED.

Aylwin, U. (1992). La pédagogie différenciée fait son entrée au collège. Pédagogie collégiale, 5(3), 30-37 Disponible en: http://aqpc.qc.ca/sites/default/files/revue/aylwin_ulric_05_3.pdf

Bergeron, L. (2015). La planification de l'enseignement a priori en fonction de la diversité des élèves. Une logique préventive et proactive. In N. Rosseau (dir.), La pédagogie de l'inclusion scolaire: Un défi ambitieux et stimulant (pp.375-397). Québec, Canada: Presses de l'Université du Québec.

Bernal, J.L. (2007). El tiempo como recurso educativo. Aula de Innovación Educativa. 163164, 38-40. Recuperado de: http://didac.unizar.es/jlbernal/articulos_propios/pdf/03_ tiemporecurso.pdf

Booth, T. \& Ainscow. M. (2011). Index for inclusion. Developing learning and participation in schools. Bristol: CSIE.

Cabedo, A. (2014a). La música como proyecto socioeducativo. En A. Giráldez, (coord.). Didáctica de la música en primaria (pp.101-122). Madrid, España: Síntesis.

Cabedo, A. (2014b). La música y su aprendizaje en la construcción de culturas para hacer las paces. Cultura de Paz, 20(64), 10-16. Recuperado de http://repositori.uji.es/xmlui/ handle/10234/124664

Caron, J. (2003). Apprivoiser les différences: guide sur la différenciation des apprentissages et la gestion des cycles. Montréal: Éditions de la Chenelière.

Epstein, J. L. (2001) School, family, and community partnerships: preparing educators and improving schools. Colorado: Westview Press.

Gil, M. (2017). Educar es un arte, LÓVA también. Peonza: Revista de literatura infantil y juvenil, 121, 46-52. Recuperado de https://issuu.com/revistapeonza/docs/peonza_121

Johnson, D.W., Johnson, R. y Holubec, E.J. (1999). El aprendizaje cooperativo en el aula. Barcelona, España: Paidós.

Jorba, J. y Cassellas, E. (1997). Estrategias y técnicas para la gestión social del aula. Vol. I La regulación y autorregulación de los aprendizajes. Madrid, España: Síntesis.

Joy, S. (2002). Original student opera: history, theory, and practice of a multi-disciplinary arts education program. (Doctoral dissertation). University of Houston.

Kahn, S. (2010). Pédagogie différenciée. Bruxelles, Belgique: De Boeck.

Kent, G. (2008). Unpeaceful music. En O. Urbain (Ed.). Music and conflict transformation: Harmonies and Dissonances in Geopolitics (pp. 104-111). London-Nueva York: I.B. Taurus.

Kline, B. (2015). Content, Process, and Product: Modeling Differentiated Instruction. Kappa Delta Pi Record 51 (1), 13-17. Recuperado de https://www.tandfonline.com/doi/abs/10.1 080/00228958.2015.988559

Leroux, M. et Paré, M. (2016). Mieux répondre aux besoins diversifiés de tous les élèves. Des pistes pour différencier, adapter et modifier son enseignement. Montréal, Canada: Chenelière éducation.

Levy, H.M. (2008). Meeting the needs of all students through differentiated instruction: helping every child reach and exceed standards. Clearing House: A Journal of Educational Strategies, Issues and Ideas, 81(4), 161-164 Recuperado de https://www.tandfonline. com/doi/abs/10.3200/TCHS.81.4.161-164

Llopis, E., Roselló, E. y Villarroya, J. (2009). "Fills de Kassim" un musical para educar en la convivencia cultural. Eufonía: Didáctica de la música, 47, 104-116.

Masip, M. y Rigol, A. (2000). El aula, escenario de la diversidad. En M.M. Aldamiz- Echevarría y otros: Cómo hacerlo. Propuestas para educar en la diversidad (pp.13-35). Barcelona: Graó.

Moriña, A. (2008). La escuela de la diversidad. Madrid, España: Síntesis. 
Okuda, M. y Gómez-Restrepo, C., (2005). Métodos en investigación cualitativa: triangulación. Revista Colombiana de Psiquiatría. XXXIV(1), 118-124. Recuperado de http://www. redalyc.org/pdf/806/80628403009.pdf

Pérez-Aldeguer, S. (2010). Inclusión social en el aula a través de la música. En: M. Sanchiz, M. Marti y I. Cremades, (Ed.), Orientación e intervención educativa. Retos para los orientadores del S. XXI (pp.637-647), Valencia, España: Tirant lo Blanch.

Pérez, J.E. y del Basto, L.M. (2011). Formación ciudadana por medio de la música andina colombiana: hacia la consolidación del sentido de pertenencia y la identidad cultural. Revista Perspectivas Educativas, 4, 241-261. Recuperado de http://revistas.ut.edu.co/ index.php/perspectivasedu/article/view/717/560

Pérez-Aldeguer, S. (2013). Efectos del Teatro Musical Colaborativo sobre el Desarrollo de la Competencia Social. Electronic Journal of Research in Educational Psychology, 11(1), 117-138 Recuperado de http://www.redalyc.org/articulo.oa?id=293125761006

Perrenoud, P. (1996). La pédagogie à l'école des différences. Paris, Francia: EFS.

Prud'homme, L., Dolbec, A. et Guay, M.-E. (2011). Le sens construit autour de la différenciation pédagogique dans le cadre d'une recherche-actionformation. Éducation et francophonie, XXXIX (2), 165-188. Recuperado de https://www.erudit.org/fr/revues/ ef/2011-v39-n2-ef05/1007733ar/

Prud'homme, L., LeBlanc, M. et Paré, M. (2013). Grille visant à soutenir l'observation en salle de classe: étude multicas de pratiques de différenciation pédagogique au Nouveau-Brunswick, au Québec et en Ontario. (Document inédit). Université du Québec à Trois-Rivières.

Prud'homme, L., Vienneau, R., Ramel, S. et Rousseau, N. (2011). La légitimité de la diversité en éducation: réflexion sur l'inclusion. Valorisation de la diversité en éducation: défis contemporains et pistes d'action. Éducation et francophonie, XXXIX(2), 6-22. Recuperado de https://www.erudit.org/fr/revues/ef/2011-v39-n2-ef05/1007725ar/

Pujolàs, P. (2008). 9 ideas clave. El aprendizaje cooperativo. Barcelona, España: Graó

RealDecreto 126/2014, de 28 de febrero, por el que se establece el currículo básico de la Educación Primaria. Recuperado de https://www.boe.es/buscar/act.php?id=BOE-A-2014-2222

Reeve, J. (1996). Motivating others: Nurturing inner motivational resources. Boston: Allyn \& Bacon.

Rojas, S. y Haya, I. (2018). Fundamentos pedagógicos de atención a la diversidad. Santander, España: Textos Universitarios, $\mathrm{n}^{\mathrm{0}} 27 .:$ Editorial Universidad Cantabria.

Ruiz-Olabuénaga, J.I. (2012). Metodología de la investigación cualitativa. Bilbao, España: Deusto.

Sanahuja, A. (2017). Diferenciación pedagógica y participación democrática en el aula inclusiva: estudio de casos múltiples (Tesis Doctoral). Universitat Jaume I, Castellón de la Plana.

Sandín. M.P. (2003). Investigación Cualitativa en Educación. Fundamentos y Tradiciones. Madrid, España: McGraw Hill.

Sarmiento, P. (2012a). La Ópera, un Vehículo de Aprendizaje (LÓVA). Eufonía Didáctica de la Música, 55, 40-47. Recuperado de https://www.grao.com/es/producto/la-opera-unvehiculo-de-aprendizaje-lova

Sarmiento, P. (2012b). El empoderamiento según LÓVA. Aula de Innovación Educativa, 213-214, 32-37. Recuperado de https://www.grao.com/es/producto/el-empoderamiento-segun-lova

Simons, H. (2011). El estudio de caso: Teoría y práctica. Madrid, España: Morata.

Stainback, S. y Stainback, W. (1999). Aulas inclusivas. Madrid, España: Narcea.

Stainback, S., Stainback, W. y Jackson, H. (1999). Hacia las aulas inclusivas. En S. Stainback y W. Stainback (eds), Aulas inclusivas (pp.21-35). Madrid, España: Narcea.

Stake, R.E. (1998). Investigación con estudio de casos. Madrid, España: Morata. 
Tomlinson, C.A. (2005). Estrategias para trabajar con la diversidad en el aula. Buenos Aires, Argentina: Paidós.

Tomlinson, C.A. (2008). El aula diversificada. Dar respuesta a las necesidades de todos los estudiantes. Barcelona, España: Octaedro.

Ugarte, D. (2007). El poder de las redes. Manual para personas, colectivos y empresas abocadas al ciberperiodismo. Madrid: Ediciones El Cobre.

UNESCO (2005). Guidelines for inclusion: Ensuring Access to Education for All. París, Francia: UNESCO.

Wan, S. W-Y. (2017). Differentiated instruction: are Hong Kong in-service teachers ready? Teachers and Teaching. Theory and Practice, 23(3), 284-311. Recuperado de https:// www.tandfonline.com/doi/abs/10.1080/13540602.2016.1204289?journalCode=ctat20 